\title{
Respiratory clinical guidelines inform ward-based nurses' clinical skills and knowledge required for evidence-based care
}

Respiratory clinical guidelines provide clinicians with evidence-based guidance for practice. Clinical guidelines also provide an opportunity to identify the knowledge and technical and non-technical skills required by respiratory ward-based registered nurses. The aim of this review was to use a systematic process to establish the core technical and non-technical skills and knowledge identified in evidence-based clinical guidelines that enable the care of hospitalised adult respiratory patients.

17 guidelines were identified in our systematic review. The quality assessment demonstrated variability in these guidelines. Common core knowledge and technical and non-technical skills were identified. These include pathophysiology, understanding of physiological measurements and monitoring, education, counselling, and ward and patient management.

The knowledge and skills extracted from respiratory clinical guidelines may inform a curriculum for ward-based respiratory nursing to ensure optimal care of adult patients.

@ERSpublications

Knowledge and skills identified in clinical guidelines provide a foundation for a curriculum for respiratory nurses http://ow.ly/w6TE30105Ya

\section{Introduction}

Respiratory medical conditions contribute to significant levels of physical, social and economic burden experienced by patients and their families $[1,2]$. Many patients with respiratory diseases may have episodes of worsening disease due to a variety of causes such as infection or responses to allergens [1]. Often these episodes require patients to be hospitalised and received ward-based therapy and care to the standard that is advocated in clinical guidelines and statements.

Evidence-based guidelines and statements for various respiratory conditions are recommended to health professionals as a summary of the evidence for treatment and therapies to ensure optimal patient outcomes. At an acute hospital ward level, clinical guidelines and statements could underpin the core clinical knowledge and skills required by registered nurses to deliver best
Cite as: Johnson AM Smith SMS. Respiratory clinical guidelines inform ward-based nurses' clinical skills and knowledge required for evidence-based care. Breathe 2016; 12: 257-266. 
practice in specialised respiratory wards. There is substantial evidence from across Europe that degree level education and staffing levels that incorporate staff-patient ratios are associated with a reduction in mortality [3]. From a specialist ward perspective, there has been limited attention given to the expectations in terms of knowledge and skills of registered nurses who provide care to respiratory patients and are not in advanced practice roles such as clinical nurse specialists and clinical nurse consultants.

Health professional societies have used consensus methodology to map clinical knowledge and skills as a process to develop ongoing educational and training activities [4]. It should be noted that the teaching and learning philosophy is not often derived during the consensus of core knowledge and skills that form a curriculum [4]. Consensus for such clinical training curriculums is often gained through expert opinion using surveys and modified Delphi methods such as e-Delphi [5] An alternative approach to consensus by experts is the examination of evidence-based respiratory clinical guidelines to ascertain the knowledge and skills required for ward-based registered nurses to be able to deliver optimal care, which may not have been considered to date.

This review reports the exploration of evidence based-respiratory clinical guidelines as a frame of reference for the essential clinical skills and knowledge required at ward level to ensure registered nurses are adequately prepared to provide the highest level of clinical care to adult patients. The aim of the review was to use a systematic process to establish the core technical and non-technical skills and knowledge espoused in respiratory evidence-based clinical guidelines that enable the care of hospitalised respiratory adult patients.

\section{Methods}

The review's objective was to identify key clinically relevant technical and non-technical skills and knowledge within evidence-based respiratory clinical guidelines. Clinical guidelines that reflect the most common respiratory conditions and specific clinical therapies associated with the care of ward-based adult patients were identified. These guidelines comprise asthma, chronic obstructive pulmonary disease (COPD), tuberculosis, lung cancer, pneumonia, influenza, smoking cessation, pulmonary rehabilitation and oxygen therapy.

\section{Search strategy}

A systematic search of respiratory clinical guidelines for acute hospital ward-based care was performed in accordance with the preferred reporting items for systematic reviews and meta-analyses (PRISMA) [6]. An a priori protocol was developed and implemented reflecting PRISMA guidance. From July 2015 to May 2016 we searched the Cochrane Library, MEDLINE, PubMed, CINAHL and Embase electronic databases. In addition, we searched the National Institute of Clinical Excellence (UK), British Thoracic Society, Thoracic Society of Australia and New Zealand, European Respiratory Society, American Thoracic Society, and the Asian Pacific Society of Respirology websites for clinical guidelines relevant to the common respiratory conditions treated in acute hospitals and the use of specific disease-related therapies. The search terms guideline, asthma, chronic obstructive pulmonary disease, community acquired pneumonia, lung cancer, influenza, oxygen therapy, smoking cessation and pulmonary rehabilitation were combined and examples of abbreviations used include guide*, COPD, pneumon*, $\mathrm{O}_{2}{ }^{*}$ oxygen, flu* influenza, pul* rehab*, smok* quit. Search limits included clinical guidelines published in the English language and the publication date was restricted to the past 7 years.

\section{Eligibility criteria}

Published evidence-based clinical guidelines that reflect the most common respiratory conditions and related therapies associated with wardbased respiratory patients' care were identified. Statements, local guidelines and clinical protocols that had or had not been published as an evidence-based document were excluded.

\section{Systematic review selection and data extraction}

Both authors independently reviewed clinical guideline titles associated with hospitalised adult respiratory patients' care prior to the full guideline being obtained. Any disagreements were discussed until consensus for inclusion or exclusion was achieved. Data were extracted from each guideline and any duplicate data noted.

\section{Evidence-based clinical guideline quality assessment}

The Appraisal of Guidelines for Research and Evaluation (AGREE) version two [7] was utilised to assess the overall quality of the published evidencebased clinical guidelines. The AGREE II instrument has 23 items in six domains that relate to the quality of a guideline. These domains comprised scope and purpose, stakeholder involvement, rigour of development, clarity of presentation, applicability and editorial independence. Each guideline was assessed in each of the six domains and a summary was tabulated (table 1 ). 


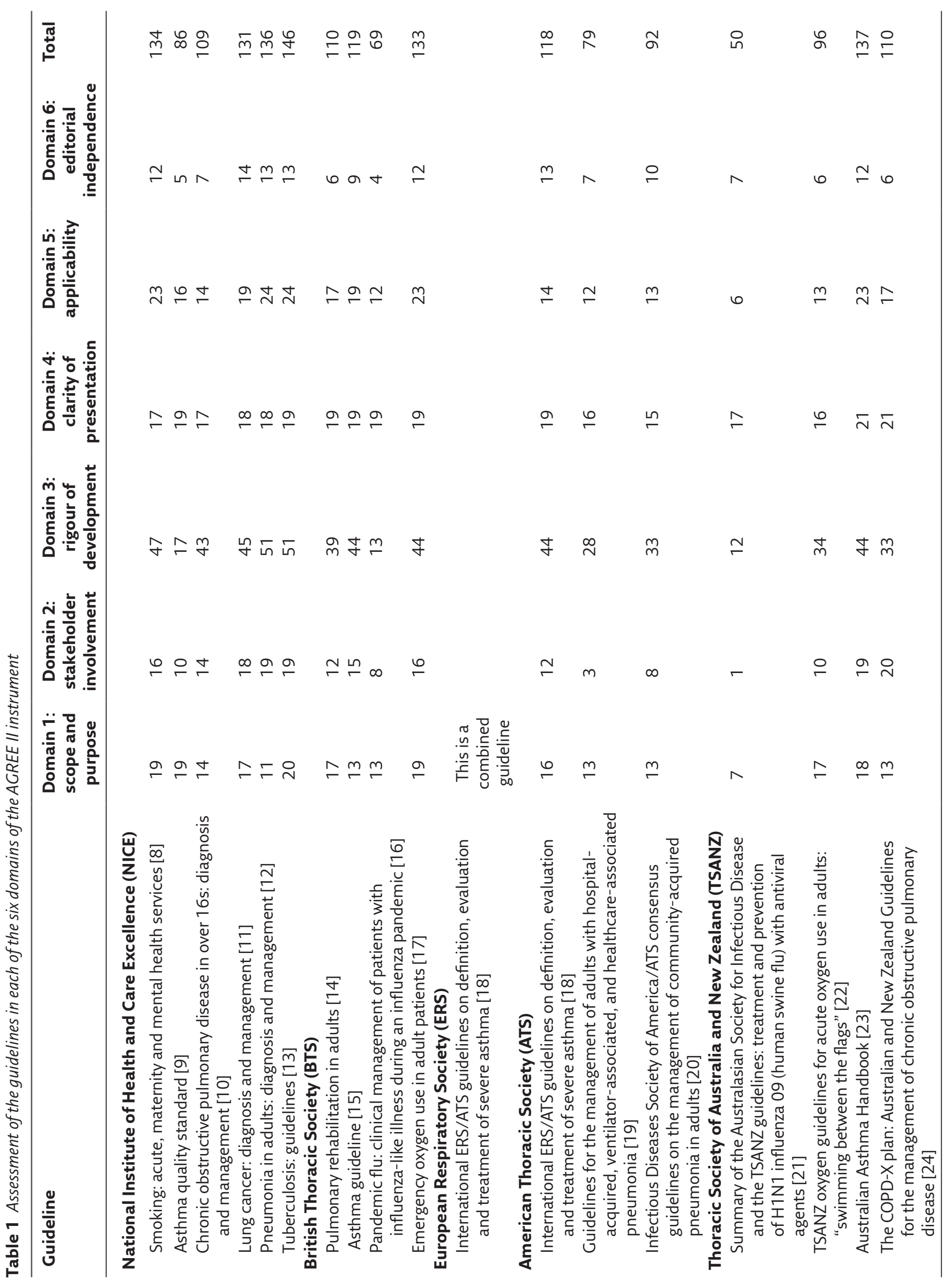




\section{Data synthesis}

Both authors confirmed the inclusion of technical and non-technical skills and knowledge specific information, displayed in table 2.

\section{Results}

The total number of relevant respiratory clinical guideline citations retrieved was 10 from the database and electronic searches. A further 19 respiratory clinical guidelines and statements were identified on respiratory society's websites. Duplicate clinical guidelines were removed and the 21 full versions of respiratory clinical guidelines were retrieved. Eligibility criteria were applied and four were considered ineligible leaving 18 respiratory clinical guidelines (figure 1) to be included in the analyses of clinical technical and non-technical skills and respiratory specific knowledge.

\section{Overview of included respiratory clinical guidelines}

18 respiratory clinical guidelines for adult care were identified and one guideline referred to two societies, ERS/ATS guidelines for severe asthma resulting in 17 guidelines. Of the 17 guidelines, the clinical diseases and therapies covered were asthma [9, 15, 18, 23], COPD [10, 24], lung cancer [11], pneumonia [12, 19, 20], tuberculosis [13], influenza [16, 21], pulmonary rehabilitation [14], oxygen therapy [17, 22], and smoking cessation [8].

\section{Quality assessment of guidelines}

Quality assessment was carried out using the AGREE II criteria, which include 23 items in six domains with a seven point rating scale for each item (table 1). The highest rating score of 161 was not achieved for any of the guidelines in this review. The tuberculosis guideline from NICE achieved a rating of 146 .

\section{Guideline-based knowledge}

The guideline-based knowledge is summarised in table 2. The core areas of knowledge within these guidelines pertain to understanding the disease and associated pathophysiology along with physiological changes, awareness of the physiological measurements for monitoring patient health status, and disease treatment associated pharmacological and non-pharmacological interventions.

\section{Technical skills}

There were common technical skills identified in the majority of guidelines. These skills included assessing the physiological status of the patient through pulse oximetry, respiratory rate, heart rate, lung function using spirometry and peak flow tools, oxygen delivery devices, arterial blood gases, sputum assessment, and administration of intravenous and drug therapy.

\section{Non-technical skills}

The non-technical skills relate to the delivery of care planning and education support. Therefore the core areas in the guidelines reviewed that are patient focused involve: understanding teaching and learning techniques; counselling patients to change behaviour; and organisational and management skills for the delivery of care. In addition to patient related non-technical skills, this review found that professional development of wardbased nurses may include training associated with the implementation of new respiratory monitoring equipment and new standards for practice in clinical guidelines.

\section{Discussion}

This review of respiratory clinical guidelines identified 17 guidelines on respiratory conditions that form the majority of patient care in respiratory wards. There were several core areas of knowledge, technical topics and non-technical subjects that were common to many of the guidelines. The use of the AGREE II criteria demonstrates that within the guidelines associated with respiratory conditions there was variability in quality. This variability may be due to extensive evidence being distilled to provide more easily accessible and readable information for clinicians who use this guidance in their everyday clinical practice.

In terms of respiratory knowledge, core knowledge was found across several clinical guidelines. These knowledge areas comprise pathophysiology, treatments and interventions, and understanding of health status measurement. Technical skills contained within these guidelines were respiratory and haemodynamic monitoring, delivery of respiratory based interventions, and administration of intravenous and other therapies. The core non-technical skills that were identified included several key topics, of which some were patient focused and others pertained to nursing staff professional development.

This respiratory knowledge and skills could be the basis for education and skill development training for ward-based nurses to reduce any inconsistencies in the delivery of bedside care to the patient. Of equal importance is the maintenance of ward-based nurses' ability to carry out technical and non-technical skills through regular opportunities to update their knowledge of the latest evidence associated with their ward-based practice. 


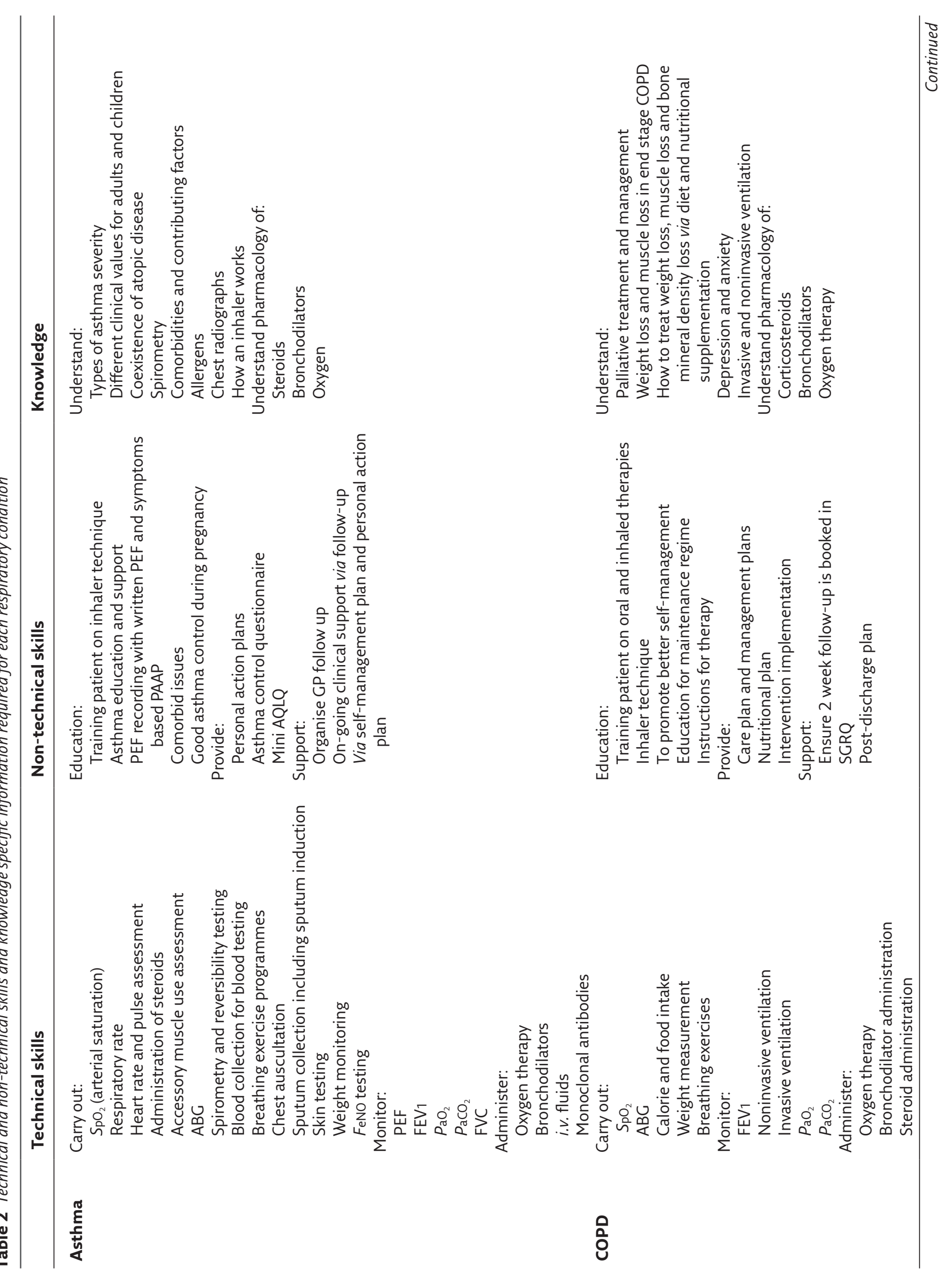




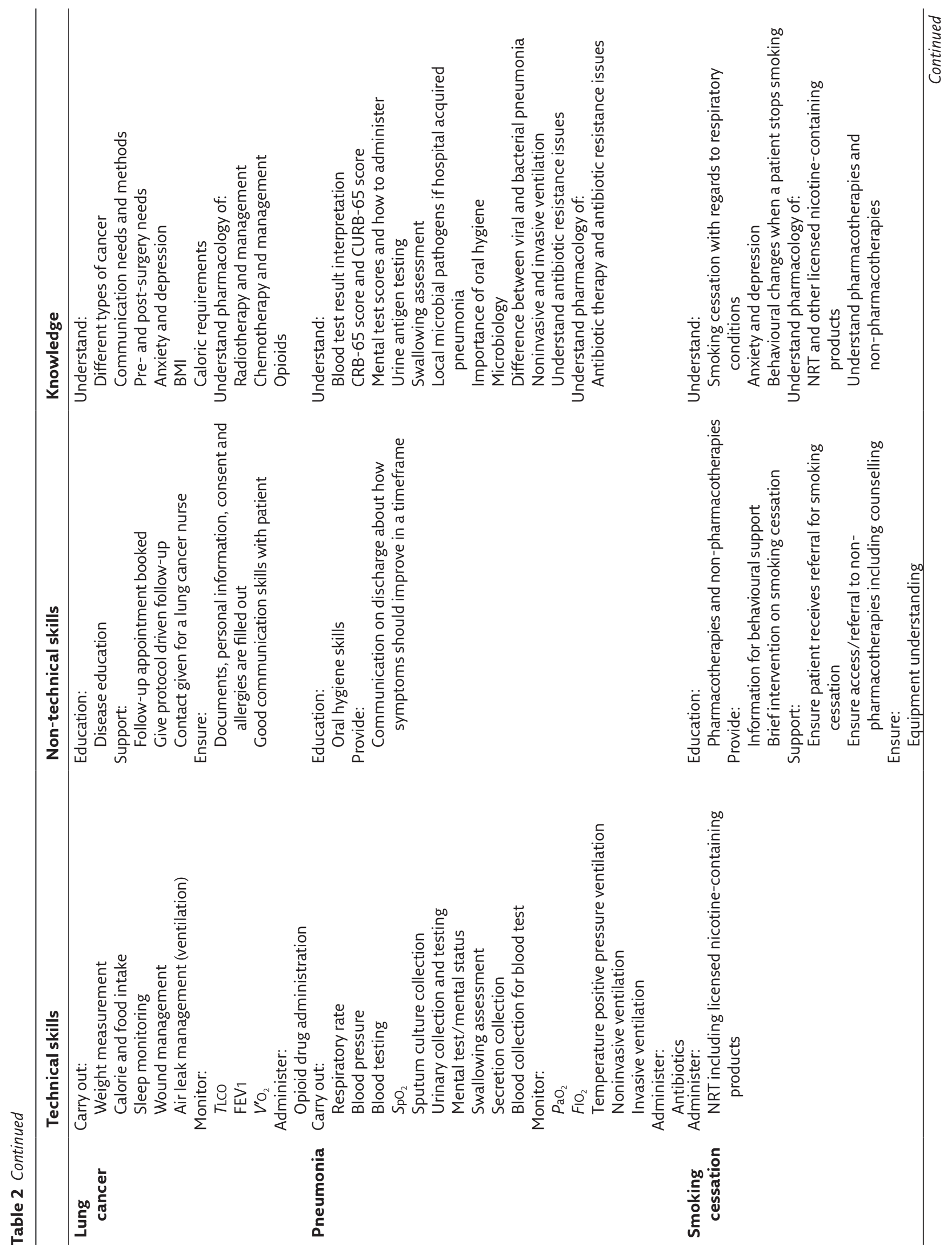




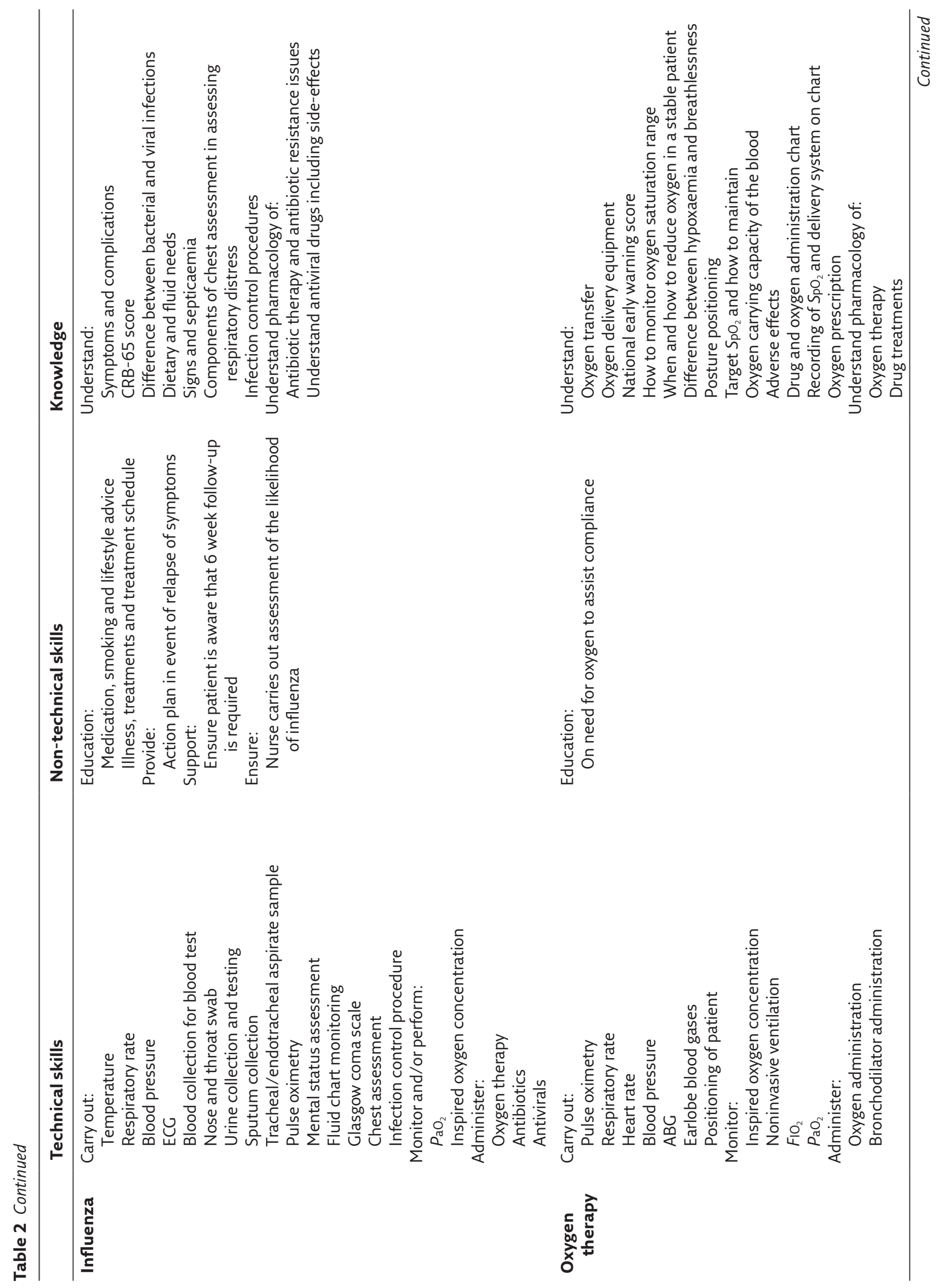




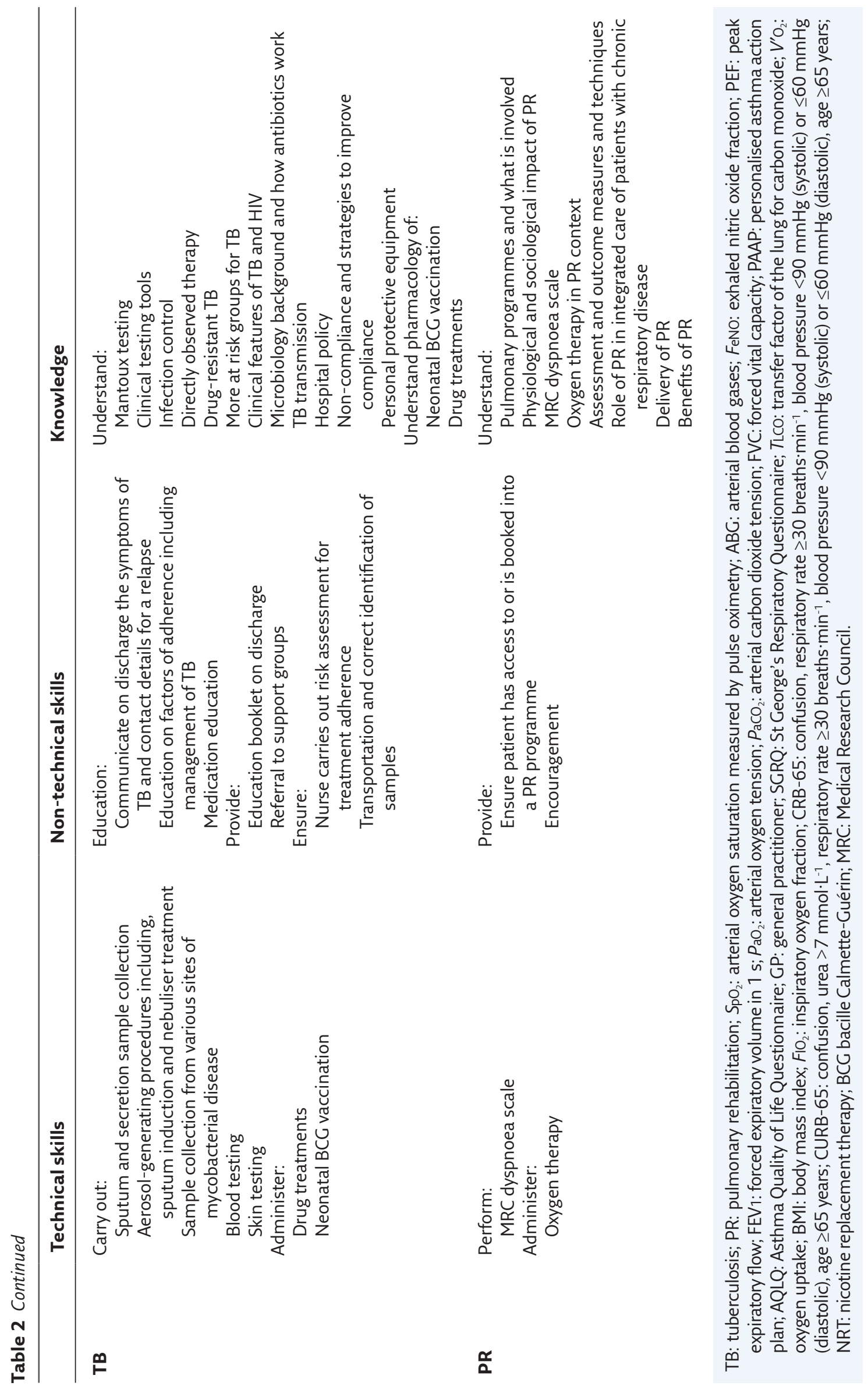


A limitation of this review is that respiratory patients often have a number of comorbid conditions and they may require additional therapies such as noninvasive ventilation. These additional medical conditions, such as heart failure, sleep apnoea and diabetes along with symptom clusters such as breathlessness, pain and fatigue, may need to be mapped across the domains of knowledge, technical and non-technical skills to ensure comprehensive clinical care is available to adult respiratory patients. Another limitation of this review is that some guidelines may be either under review or were not identified during our search. Using the process outlined in this review, additional information from new guidelines could be mapped in a similar way in terms of respiratory practice knowledge and skills. A replication of this mapping process may be used for nurses who provide care for paediatric respiratory patients as a way to highlight their speciality ward-based knowledge and skills that represent their professional practice.

While international guidance for respiratory conditions aims ultimately to provide the best available evidence, various countries will have a different scope of practice for different disciplines. The information in this review needs to be considered in terms of each countries nurses' industrial award change to conditions of employment and registered nurses scope of ward-level practice.

\section{Conclusion}

This review sought to outline the knowledge, technical and non-technical skills required to delivery optimal respiratory evidence-based guideline care. The core knowledge required by nurses spanned several guidelines and consisted of disease pathophysiology and pharmacological and non-pharmaceutical interventions. The technical skills promoted within these guidelines were respiratory and haemodynamic monitoring,

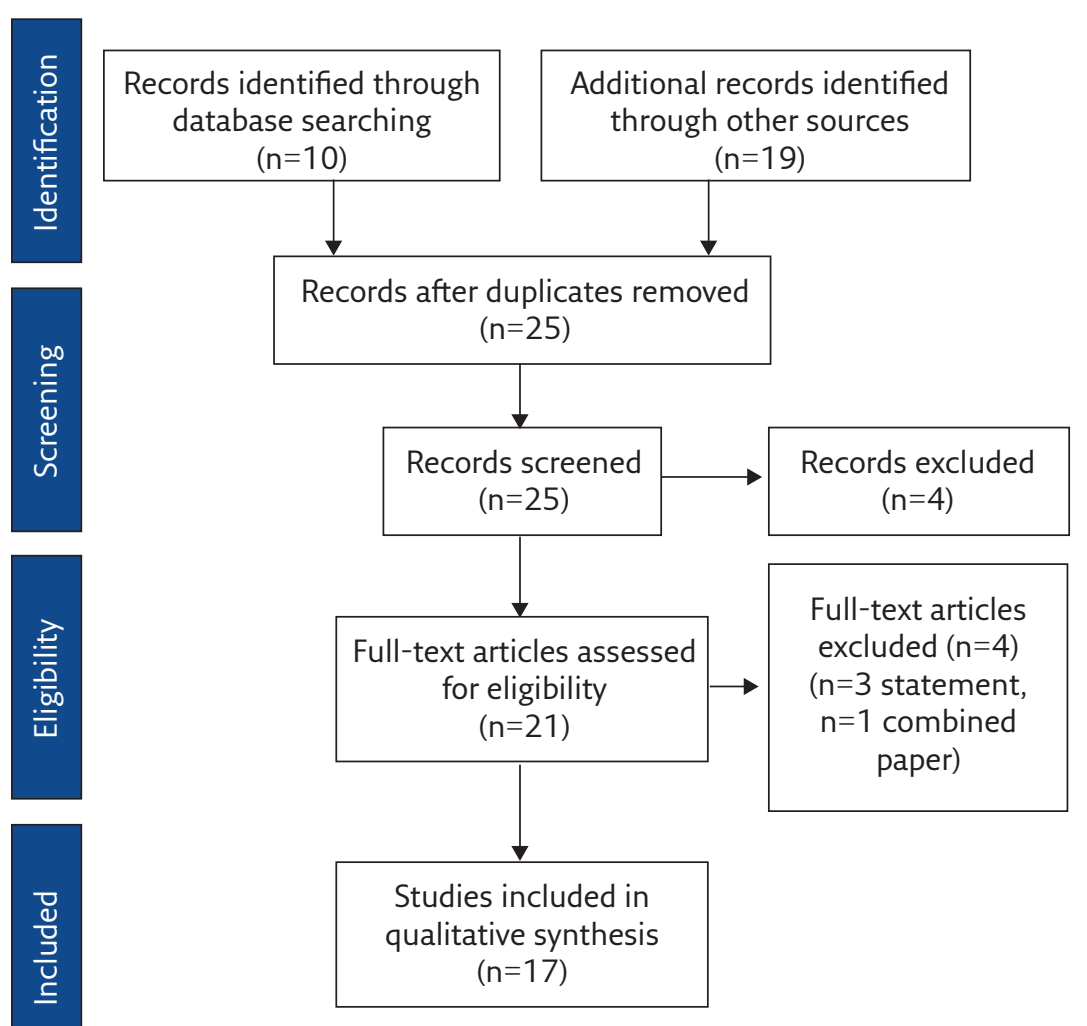

Figure 1 The process of selection of respiratory clinical guidelines performed in accordance with the PRISMA statement [6]. Adapted from [6].

delivery of respiratory based interventions and administration of intravenous therapy. In addition the non-technical skills were applicable to both patient-focused care and ward-based nursing staff professional development. While a limitation of this review may be that some guidelines were not identified, this process provides a foundation for building a professional curriculum to support the education and development of respiratory ward-based nurses to ensure the delivery of optimal patient care.

\section{Conflict of interest}

None declared.

\section{References}

1. Forum of International Respiratory Societies. Respiratory Disease in the World: Realities of Today - Opportunities for Tomorrow. Sheffield, European Respiratory Society, 2013.

2. Vestbo J, Hurd SS, Agustí AG, et al. Global strategy for the diagnosis, management, and prevention of chronic obstructive pulmonary disease. Am J Respir Crit Care Med 2013; 187: 347-365.

3. Aiken LH, Sloane DM, Bruynel L, et al. Nurse staffing and education and hospital mortality in nine European countries: a retrospective observational study. Lancet 2014; 383 : 1824-1830.
4. Astin F, Carroll D, De Geest S, et al. A core curriculum for the continuing professional development of nurses working in cardiovascular settings: development by the education committee of the Council on Cardiovascular Nursing and Allied Health Professions (CCNAP) on behalf of the European Society of Cardiology. EurJ Cardiovasc Nurs 2015; 14: Suppl., S1-S17.

5. Jones J, Hunter D. Consensus methods for medical and health services research. BMJ 1995; 311: 376-380.

6. Moher D, Liberati A, TetzlaffJ, et al. Preferred reporting items for systematic reviews and meta-analyses: the PRISMA statement. PLoS Med 2009; 6: e1000097. 
7. Brouwers M, Kho ME, Browman GP, et al. AGREE II: advancing guideline development, reporting and evaluation in healthcare. CMAJ 2010; 182: E839-E842.

8. National Institute for Health and Care Excellence. Smoking: acute, maternity and mental health services. NICE guidelines [PH48]. www.nice.org.uk/guidance/ph48 Date last updated: November 2013. Date last accessed: May 15, 2016.

9. National Institute for Health and Care Excellence. Asthma NICE quality standard [QS25]. www.nice.org.uk/guidance/qs25 Date last updated: February 2013. Date last accessed: May 15, 2016

10. National Institute for Health and Care Excellence. Chronic obstructive pulmonary disease in over 16s: diagnosis and management. NICE guidelines [CG101]. www.nice.org. uk/guidance/cg101/ Date last updated: June 2010. Date last accessed: May 15, 2016.

11. National Institute for Health and Care Excellence. Lung cancer: diagnosis and management. NICE guidelines [CG121]. www.nice.org.uk/guidance/cg121 Date last updated: April 2011. Date last accessed: May 15, 2016.

12. National Institute for Health and Care Excellence. Pneumonia in adults: diagnosis and management. NICE guidelines [CG191]. www.nice.org.uk/guidance/cg191 Date last updated: December 2014. Date last accessed: May 15, 2016.

13. National Institute for Health and Care Excellence. Tuberculosis NICE guidelines [NG33]. www.nice.org.uk/ guidance/ng33 Date last updated: December 2014. Date last accessed: May 15, 2016

14. Bolton CE, Bevan-Smith EF, Blakey JD, et al. British Thoracic Society guideline on pulmonary rehabilitation in adults. Thorax 2013; 68: Suppl. 2, ii1-ii30.

15. British Thoracic Society. Asthma Guideline. www. brit-thoracic.org.uk/guidelines-and-quality-standards/ asthma-guideline/ Date last accessed May 15, 2016.

16. British Infection Society, British Thoracic Society, Health Protection Agency. Pandemic flu: clinical management of patients with an influenza-like illness during an influenza pandemic. Provisional guidelines from the British
Infection Society, British Thoracic Society, and Health Protection Agency in collaboration with the Department of Health. Thorax 2007; 62: Suppl. 1, 1-46.

17. O'Driscoll BR, Howard LS, Davidson AG. BTS guideline for emergency oxygen use in adult patients. Thorax 2008; 63: Suppl. 6, vi1-vi30.

18. Chung KF, Wenzel SE, Brozek JL, et al. International ERS/ATS guidelines on definition, evaluation and treatment of severe asthma. Eur RespirJ 2014; 43: 343-373.

19. American Thoracic Society, Infectious Diseases Society of America. Guidelines for the management of adults with hospital-acquired, ventilator-associated, and healthcareassociated pneumonia. Am J Respir Crit Care Med 2005; 171: 388-416.

20. Mandell LA, Wunderink RG, Anzueto A, et al. Infectious Diseases Society of America/American Thoracic Society consensus guidelines on the management of community-acquired pneumonia in adults. Clin Infect Dis 2007; 44: Suppl., S27-S72.

21. Cheng AC, Dwyer DE, Kotsimbos AT, et al. Summary of the Australasian Society for Infectious Diseases and the Thoracic Society of Australia and New Zealand guidelines: treatment and prevention of H1N1 influenza 09 (human swine influenza) with antiviral agents. Med J Aust 2009; 191: 142-145.

22. Beasley R, Chien J, Douglas J, et al. Thoracic Society of Australia and New Zealand oxygen guidelines for acute oxygen use in adults: 'Swimming between the flags'. Respirology 2015; 20: 1182-1191.

23. National Asthma Council Australia. Australian Asthma Handbook. www.nationalasthma.org.au/handbook Date last accessed: May 15, 2016.

24. Abramson M, Crockett AJ, Dabscheck E, et al. The COPD-X Plan: Australian and New Zealand Guidelines for the management of Chronic Obstructive Pulmonary Disease. http://copdx.org.au/copd-x-plan/ Date last updated: March 2016. Date last accessed: May 15, 2016. 